\title{
MONITORAMENTO DE ATIVOS HOSPITALARES ATRAVÉS DE UMA REDE DE SENSORES SEM FIO
}

\begin{abstract}
Álvaro Medeiros Avelino
Mestre pelo Programa de Pós-Graduação em Engenharia Elétrica e de Computação, da Universidade Federal do Rio Grande do Norte, 2009. Atualmente é professor do ensino básico, técnico e tecnológico do campus Ipanguaçu do Instituto Federal de Educação

Tecnológica do Rio Grande do Norte. alvaro.medeiros@ifrn.edu.br

Samuel de Paiva Rêgo

Graduação em Engenharia de Computação pela Universidade Federal do Rio Grande do Norte, 2011. Atualmente é professor substituto do campus Natal-Central do Instituto Federal de Educação Tecnológica do Rio Grande do Norte. Samuel.rego@ifrn.edu.br

\section{Leonardo Gomes de Paiva Amorim}

Mestre pelo Programa de Pós-Graduação em Engenharia Elétrica e de Computação, da Universidade Federal do Rio Grande do Norte, 2011. Atualmente é professor do ensino básico, técnico e tecnológico do campus Santa Cruz do Instituto Federal de Educação

Tecnológica do Rio Grande do Norte. leonardo.amorim@ifrn.edu.br Jefferson Doolan Fernandes

Mestre pelo Programa de Pós-Graduação em Ciência e Engenharia de Petróleo, da Universidade Federal do Rio Grande do Norte, 2010. Atualmente é professor do ensino básico, técnico e tecnológico do campus Santa Cruz do Instituto Federal de Educação Tecnológica do Rio Grande do Norte. jefferson.fernandes@ifrn.edu.br
\end{abstract}

\section{RESUMO}

Este trabalho propõe um sistema de monitoramento em tempo real de ativos hospitalares em que os seus nós (ou sensores) comunicam-se entre si utilizando a tecnologia ZigBee. $\mathrm{O}$ sistema é composto por vários sensores (escravos) que periodicamente monitoram a temperatura do local onde estão abrigados e enviam essa informação ao mestre da rede sem fio. Este, por sua vez, além da comunicação ZigBee, possui também interface Ethernet, disponibilizando, desta maneira, a visualização das temperaturas na rede cabeada do hospital.

PALAVRAS-CHAVE: Redes de Sensores sem Fio, ZigBee, sistema de tempo real, automação, economia de energia.

\section{MEDICINE TEMPERATURE MONITORING USING WIRELESS SENSOR NETWORKS}

\begin{abstract}
This work proposes a real time monitoring system applied to medicine stored in hospitals. This system is disposed as a wireless sensor network, which uses ZigBee technology. It is composed by many slaves which periodically monitor local temperature and send it to the master. The last, besides ZigBee master, has also Ethernet interface, making available, this way, previewing the temperatures through the hospital network.
\end{abstract}


KEYWORDS: Wireless Sensor Networks, ZigBee, real time system, automation, energy saving.

\section{MONITORAMENTO DE ATIVOS HOSPITALARES ATRAVÉS DE UMA REDE DE SENSORES SEM FIO}

\section{INTRODUÇÃO}

Atualmente qualquer hospital (independente da dimensão do mesmo) armazena medicamentos de alto custo e que necessitam ser armazenados em uma determinada faixa de temperatura (5 a $15{ }^{\circ} \mathrm{C}$, por exemplo). Como monitorar esta temperatura de forma confiável? Se tal monitoramento não for realizado de maneira periódica e sistemática e a temperatura variar além ou aquém da faixa estabelecida pelo fabricante o produto tornarse-á inutilizável.

Como isso é feito atualmente? Ao visitar um hospital privado na cidade do Natal constatou-se que essa verificação é feita diariamente (uma vez por dia) por um funcionário do hospital, que preenche uma tabela com a identificação da geladeira, temperatura observada no momento da checagem e o horário em que a verificação ocorreu. Em cada geladeira há um termômetro digital, conforme o exibido na Figura 1.

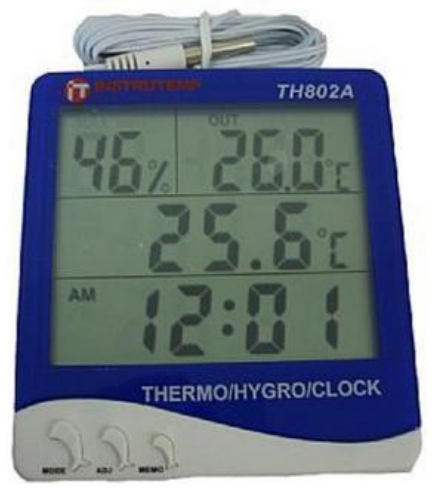

Figura 1. Termômetro digital.

A desvantagem dessa abordagem é que uma variação na temperatura pode ocorrer logo após a verificação ter sido feita. No caso do exemplo citado no parágrafo anterior a alteração só seria percebida pelo funcionário durante a medição do dia seguinte.

A automação é uma ferramenta que agrega valor e segurança a um processo que antes era manual (como o caso citado nos dois parágrafos anteriores). A automação mais tradicional que se conhece é a industrial (FERNANDES, 2010), porém ao longo dos últimos anos ela tem se expandido para diversas áreas, tais como:

- Militar: guerra eletrônica, míssil, torpedo

- Residencial: monitoramento através de câmeras, alarme, portão eletrônico

- Hospitalar: oxímetro de pulso (BRANDÃO, 1996), equipamentos de imagens médicas (ultrassonografia, ecocardiograma)

- Comercial: sistema de emissão de nota fiscal, controle de estoque

- Veicular: vidro elétrico, trava elétrica, ABS, Air Bag 
A automação hospitalar vai desde equipamentos utilizados durante procedimentos médicos até o sistema que o hospital utiliza com o cadastro dos pacientes. Trata-se de uma área em plena expansão atualmente.

No Hospital Israelita Albert Einstein, por exemplo, foi implantada uma solução que utiliza etiquetas RFID e a rede Wi-Fi do hospital para a propagação dos dados. Entretanto, a solução proposta utiliza mais de 1.000 Access Points Wi-Fi, o que torna a solução pouco atraente para hospitais de pequeno e médio porte, tendo em vista que a maioria deles nem dispõe de Wi-Fi em todo o hospital (PERIN, 2012).

Uma maneira de projetar uma solução automatizada é utilizando sistemas embarcados, que consiste de hardware e software, podendo conter algumas partes mecânicas adicionais (AVELINO, 2009). É comum que algumas aplicações de sistemas embarcados tenham restrições de consumo de energia. Um exemplo é o telefone celular: quanto maior a autonomia da bateria melhor será a impressão final do cliente.

É aí que surge a eficiência energética. Não mais é suficiente apenas projetar um sistema embarcado, mas sim é preciso pensar em como fazer o sistema gastar somente o necessário de energia (AMORIM, 2011).

A fim de propor uma solução para o problema citado este trabalho apresenta um sistema embarcado de monitoramento wireless que utiliza a tecnologia ZigBee. A próxima Seção detalha os componentes do sistema.

\section{ESPECIFICAÇÃO DO SISTEMA}

A solução proposta faz uso de uma Rede de Sensores Sem Fio (RSSF). Tal rede consiste de vários escravos, cada um atuando como um termômetro digital wireless, e um mestre, que coleta os dados dos escravos e disponibiliza na rede do hospital.

Cada escravo contém:

- Um sensor de temperatura tipo termopar

- Um microcontrolador de 8 bits da família ATXmega, da Atmel, que é um processador dedicado, responsável por coletar a temperatura do termopar e enviála ao transceiver ZigBee

- Cristal oscilador de $8 \mathrm{MHz}$, que seve como base para a contagem de tempo

- Um transceiver ZigBee modelo XBee, responsável por enviar as informações adquiridas do microcontrolador ao mestre da rede ZigBee

- Uma bateria alcalina de $9 \mathrm{~V}$.

Foi necessário criar uma placa de circuito impresso (utilizando um software de desenho de circuitos e uma máquina prototipadora) dedicada a este circuito, a fim de tornar a placa a menor possível.

O projeto do escravo contemplou abrigar a placa de circuito impresso, o transceiver ZigBee e a bateria de $9 \mathrm{~V}$. Para isso a equipe de desenvolvimento optou por utilizar uma caixa de dimensões $24 \mathrm{~mm}$ (altura) x $60 \mathrm{~mm}$ (largura) x $75 \mathrm{~mm}$ (comprimento), conforme pode ser observado na Figura 2. 


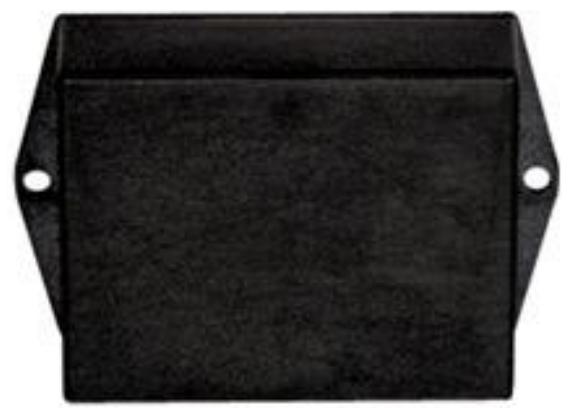

Figura 2. Caixa que abriga o escravo.

O escravo pesa menos de $100 \mathrm{~g}$ e pode satisfatoriamente ser fixado à geladeira utilizando fita dupla face, por exemplo.

O mestre consiste também de um microcontrolador, porém este possui uma capacidade superior de processamento, se comparado ao microcontrolador utilizado no escravo. A função do mestre é coletar os dados dos escravos, armazená-los em um banco de dados local e disponibilizá-los (para consulta) na rede do hospital. Para coletar os dados foi utilizado um transceiver XBee PRO, configurado como mestre da rede ZigBee. A Figura 3 mostra a placa de desenvolvimento utilizada como mestre.

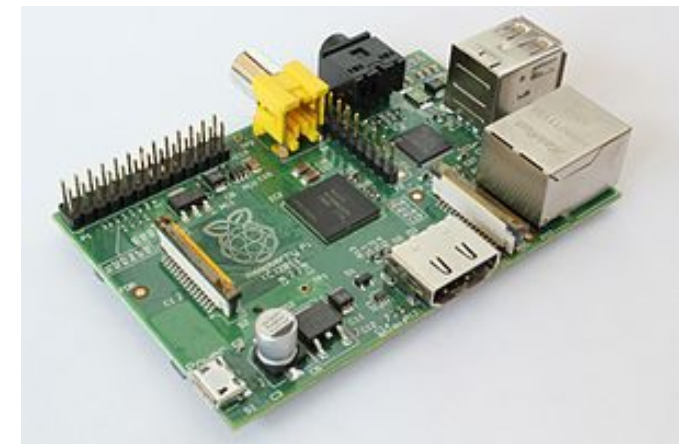

Figura 3. Raspberry Pi.

Os motivos que levaram à escolha desta placa de desenvolvimento foram as dimensões reduzidas $(86,5 \mathrm{~mm} \times 56 \mathrm{~mm}$ ), interface Ethernet, USB, HDMI (que a maioria dos concorrentes da Raspberry Pi ainda não disponibiliza) e slot para cartão SD. Nessa aplicação esse cartão é utilizado para armazenar o banco de dados contendo as temperaturas adquiridas dos sensores (escravos).

A Seção seguinte aborda o funcionamento do sistema, com foco especial no escravo da rede.

\section{FUNCIONAMENTO DO SISTEMA}

O escravo foi projetado para funcionar como um sistema de tempo real, a fim de garantir medições periódicas de temperatura. Existem basicamente dois tipos de sistema de tempo real: hard real-time e soft real-time. $\mathrm{O}$ primeiro não admite, sob hipótese alguma, atraso. $\mathrm{O}$ último admite atraso, ainda que alguma perda de performance do sistema como consequência (OSHANA, 2006). 
O escravo foi especificado para funcionar como um soft real-time, ou seja, admite algum tipo de atraso. Ou seja, para esta aplicação é indiferente se uma aquisição de temperatura ocorre a cada 30s ou a cada 31s. Outras áreas de aplicação (como aviação, por exemplo) não admitem este tipo de atraso, e, portanto, exige que o sistema seja hard real-time.

Para garantir que o sistema é de tempo real a programação do microcontrolador utilizou uma interrupção periódica, programada para ocorrer a cada $30 \mathrm{~s}$.

O microcontrolador utilizado nesta aplicação possui vários módulos de sleep. De forma geral, quanto menor o consumo menos dispositivos internos do microcontrolador estão disponíveis. Para esta aplicação é utilizado o modo Power-Save, que mantém ligado um contador de tempo que é utilizado para acordar o microcontrolador (uma espécie de alarme).

A Tabela 1 mostra o consumo de energia do microcontrolador, do transceiver XBee e XBee PRO, segundo dados fornecidos pelos fabricantes (ATMEL, 2012), (DIGI, 2012).

Tabela 1: Consumo energético dos dispositivos utilizados.

\begin{tabular}{|l|c|c|}
\hline DISPOSITIVO & MODO & CONSUMO \\
\hline Microcontrolador & Modo ativo & $12 \mathrm{~mA}$ \\
\hline Microcontrolador & Baixo consumo & $3 \mu \mathrm{A}$ \\
\hline XBee & Modo ativo & $50 \mathrm{~mA}$ \\
\hline XBee & Baixo consumo & $10 \mu \mathrm{A}$ \\
\hline XBee Pro & Modo ativo & $340 \mathrm{~mA}$ \\
\hline XBee Pro & Baixo consumo & $10 \mu \mathrm{A}$ \\
\hline
\end{tabular}

Após a inicialização, o microcontrolador fica num modo de sleep, no qual alguns periféricos são desligados e o consumo de energia é reduzido sensivelmente. A estrutura do microcontrolador responsável pela contagem dos $30 \mathrm{~s}$ e o vetor que armazena a temperatura adquirida permanecem ativos. Ao atingir esse tempo o microcontrolador retorna ao estado ativo (sai do modo de economia de energia) e realiza uma nova leitura da temperatura do termopar. Essa temperatura é armazenada num vetor e o microcontrolador volta ao estado de economia de energia (sleep). Isso se repete até a contagem de tempo atingir 10 min (20 aquisições de temperatura).

Quando isso ocorre, além de adquirir a temperatura do termopar o microcontrolador acorda o transceiver ZigBee (que antes também estava no modo de baixo consumo) para que este envie os dados de temperatura através da rede ZigBee. Após o envio tanto o microcontrolador quanto o transceiver ZigBee retornam ao modo de economia de energia. A sequência de passos pode ser visualizada na Figura 4.

Esses tempos podem ser redimensionados; portanto esta escolha foi uma decisão inicial da equipe de desenvolvimento.

De qualquer forma a ideia de utilizar o vetor é minimizar o tempo durante o qual transceiver ZigBee está no modo ativo, pois segundo a Tabela 1, é o componente do escravo que mais consome energia. 


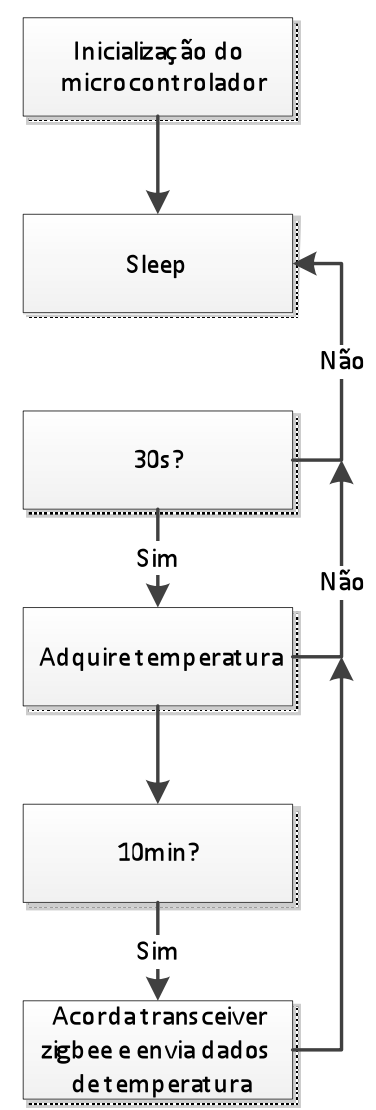

Figura 4. Fluxograma de funcionamento do escravo.

A próxima Seção discorre sobre os resultados obtidos durante a elaboração deste trabalho.

\section{RESULTADOS}

O que se pretende nesta Seção é analisar em termos de gasto de energia o quanto se conseguiu otimizar o sistema ao optar por utilizar os modos de economia de energia dos dispositivos componentes do escravo.

O ponto-chave de um sistema de tempo real é que o desenvolvedor tenha domínio total sobre o que está acontecendo a cada instante de tempo. No caso desta aplicação o que interessa é saber quanto tempo leva o processamento da interrupção de aquisição de temperatura. E aí há duas situações:

- Na primeira apenas a temperatura é adquirida e o microcontrolador volta ao modo de baixo consumo

- Na segunda, além de adquirir a temperatura, supõe que o vetor de aquisição está cheio e é preciso acordar o transceiver ZigBee para que este envie o vetor de temperaturas ao mestre

A primeira situação, do ponto de vista do tempo de processamento necessário, é bastante simples. Medições feitas com osciloscópio digital indicaram que nesse caso o tempo necessário ao processamento é de $3,51 \mathrm{~ms}$. Nesta situação o consumo de corrente gira em torno de $15 \mathrm{~mA}$.

A segunda é um pouco mais complexa. Ao acordar o transceiver ZigBee o consumo total de corrente da placa cresce significativamente (gira em torno de $70 \mathrm{~mA}$ ). Esse consumo se 
mantém até que o transceiver ZigBee retorne ao estado de baixo consumo. Ao contrário da primeira situação, em que temos um tempo definido, na segunda tem-se comunicação sem fio, que não é um evento determinístico, ou seja, é impossível afirmar com 100\% de exatidão o tempo necessário a essa comunicação. Vale salientar ainda que esta etapa depende também de uma confirmação de recebimento da mensagem pelo mestre da rede ZigBee, o que torna um pouco mais complexo determinar com exatidão o tempo necessário a essa comunicação.

Após o transceiver ZigBee retornar ao estado de baixo consumo, o microcontrolador também o faz. Medições feitas utilizando osciloscópio digital mostraram que o tempo total necessário gira em torno de $100 \mathrm{~ms}$.

Testes feitos em laboratório utilizando uma bateria de $9 \mathrm{~V}$ alcalina mostraram que se não for utilizado o modo de baixo consumo do microcontrolador e do transceiver ZigBee (ambos em modo ativo) a autonomia da bateria é de cerca de $3 \mathrm{~h}$. Ou seja, é inviável para a aplicação em questão substituir a bateria a cada $3 \mathrm{~h}$ (seria necessário uma fonte externa e, portanto, não seria uma solução totalmente sem fio).

Ao utilizar os modos de economia de energia é possível reduzir em cerca de 1.000 vezes o consumo energético do circuito. Ou seja, a autonomia da bateria passaria a cerca de 3.000 h, o que equivale a cerca de 4 meses de uso.

A Seção seguinte apresenta a conclusão do trabalho.

\section{CONCLUSÃO}

Este trabalho analisou o funcionamento do nó escravo do sistema em termos de consumo de energia. As análises feitas são aproximadas, porém são fundamentadas nos dados disponibilizados pelos fabricantes. Outros fatores não citados neste trabalho influem diretamente no consumo do circuito, tais como: largura da trilha utilizada no circuito, potência dissipada pelos resistores e frequência do cristal oscilador (quanto maior a frequência maior será o consumo).

A análise comprovou que ao utilizar os modos de economia de energia é possível otimizar o consumo energético, pois o processador é quase que desligado nos instantes em que se encontra ocioso e é mantido no modo ativo apenas quando realmente houver alguma tarefa a ser realizada.

A automação desse tipo de processo (se projetado como um sistema de tempo real) prova ser uma solução de grande confiabilidade, pois o sistema é capaz de fornecer respostas (temperaturas) periódicas e precisas. Tais informações podem ser disponibilizadas na rede local do hospital, sem a necessidade de um funcionário verificar periodicamente a temperatura de cada termômetro. É possível também gerar um alarme caso a temperatura de um determinado termômetro esteja fora da faixa especificada.

A próxima Seção sugere algumas melhorias a serem implantadas nas próximas etapas do trabalho.

\section{MELHORIAS FUTURAS}


Em uma segunda etapa do trabalho pretende-se incorporar ao microcontrolador o monitoramento da tensão da bateria de $9 \mathrm{~V}$, permitindo assim enviar através da rede ZigBee um alerta de que a bateria precisa ser substituída.

Outro teste que vale a pena ser realizado é eliminar o cristal oscilador externo e utilizar o interno. De forma geral, o externo é mais estável, mas é válido checar se o interno funciona de forma satisfatória para esta aplicação (já que se trata de um soft real-time). A utilização do oscilador interno simplifica o circuito (elimina o próprio oscilador e dois capacitores, utilizados como filtro) e reduz o consumo de energia.

Se necessário for é também possível substituir a rede ZigBee por uma Wi-Fi, porém a equipe desenvolvedora entende que trata-se de uma solução mais arrojada em termos de custo.

Por último, é possível ligar um monitor de vídeo ao mestre, a fim de visualizar as informações de temperatura em tempo real.

\section{REFERÊNCIAS BIBLIOGRÁFICAS}

1. AMORIM, L. G. de P. Utilização de Sistemas Dedicados e Protocolos de Rede Aplicados à Eficiência Energética da Iluminação Pública. Dissertação de Mestrado Universidade Federal do Rio Grande do Norte, 2011.

2. AVELINO, Á. M. Processamento embarcado aplicado a um sistema de detecção de vazamentos. Dissertação de Mestrado - Universidade Federal do Rio Grande do Norte, 2009.

3. ATMEL. ATXmega128D4 Datasheet. 2012.

4. BRANDÃO, G. B. Controlador Programável de Infusão para Ambientes Hospitalares. Dissertação de Mestrado - Universidade Federal de Pernambuco, 1996.

5. DIGI. XBee/XBee PRO RF Modules. Product Manual v1.xEx - 802.15.4 Protocol. 2012.

6. FERNANDES, J. D. Desenvolvimento de Sistemas Embarcados para Redes de Sensores e Atuadores Sem Fio Aplicadas em Unidades de Elevação de Petróleo do Tipo Plunger-Lift - Universidade Federal do Rio Grande do Norte, 2010.

7. PERIN, EDSON. Hospital Israelita Albert Einstein usa RFID para rastreamento de ativos. Disponível em brasil.rfidjournal.com/estudos_de caso/9528/3. Acessado em 26/11/2012.

8. OSHANA, R. DSP Software Development Techniques for Embedded and Real-Time Systems. Elsevier, 2006. 